Internat. J. Math. \& Math. Sci.

Vol. 24, No. 8 (2000) 519-527

S016117120000404X

(C) Hindawi Publishing Corp.

\title{
ON PARTITIONS WITH DIFFERENCE CONDITIONS
}

\author{
JOSÉ PLÍNIO O. SANTOS and PAULO MONDEK
}

(Received 26 October 1999)

\begin{abstract}
We present two general theorems having interesting special cases. From one of them we give a new proof for theorems of Gordon using a bijection and from another we have a new combinatorial interpretation associated with a theorem of Göllnitz.
\end{abstract}

Keywords and phrases. Partitions, combinatorial identities, $q$-series.

2000 Mathematics Subject Classification. Primary 11P83.

1. Introduction. Many of the identities given by Slater [5] have been used in the proofs of several combinatorial results in partitions. In this paper, we use Slater [5, equations $34,36,48,53$, and 57], listed, in this order, below

$$
\begin{aligned}
\sum_{n=0}^{\infty} \frac{\left(-q ; q^{2}\right)_{n} q^{n(n+2)}}{\left(q^{2} ; q^{2}\right)_{n}} & =\prod_{n=1}^{\infty} \frac{\left(1+q^{2 n-1}\right)\left(1-q^{8 n-1}\right)\left(1-q^{8 n-7}\right)\left(1-q^{8 n}\right)}{\left(1-q^{2 n}\right)} \\
\sum_{n=0}^{\infty} \frac{\left(-q ; q^{2}\right)_{n} q^{n^{2}}}{\left(q^{2} ; q^{2}\right)_{n}} & =\prod_{n=1}^{\infty} \frac{\left(1+q^{2 n-1}\right)\left(1-q^{8 n-3}\right)\left(1-q^{8 n-5}\right)\left(1-q^{8 n}\right)}{\left(1-q^{2 n}\right)}, \\
\prod_{n=1}^{\infty}\left(1-q^{12 n-5}\right)\left(1-q^{12 n-7}\right)\left(1-q^{12 n}\right)-q \prod_{n=1}^{\infty}\left(1-q^{12 n-1}\right)\left(1-q^{12 n-11}\right)\left(1-q^{12 n}\right) & =\prod_{n=1}^{\infty}\left(1-(-1)^{n} q^{3 n-1}\right)\left(1+(-1)^{n} q^{3 n-2}\right)\left(1-(-1)^{n} q^{3 n}\right) \\
\sum_{n=0}^{\infty} \frac{\left(q ; q^{2}\right)_{2 n} q^{4 n^{2}}}{\left(q^{4} ; q^{4}\right)_{2 n}} & =\prod_{n=1}^{\infty} \frac{\left(1-q^{12 n-5}\right)\left(1-q^{12 n-7}\right)\left(1-q^{12 n}\right)}{\left(1-q^{4 n}\right)} \\
\sum_{n=0}^{\infty} \frac{\left(-q ; q^{2}\right)_{2 n+1} q^{4 n(n+1)}}{\left(q^{4} ; q^{4}\right)_{2 n+1}} & =\prod_{n=1}^{\infty} \frac{\left(1+q^{12 n-1}\right)\left(1+q^{12 n-11}\right)\left(1-q^{12 n}\right)}{\left(1-q^{4 n}\right)}
\end{aligned}
$$

to prove some results in partitions where we use the standard notation

$$
\left.\begin{array}{l}
(a ; q)_{0}=1 \\
(a ; q)_{n}=(1-a)(1-a q) \cdots\left(1-a q^{n-1}\right), \\
(a ; q)_{\infty}=\lim _{n \rightarrow \infty}(a ; q)_{n},
\end{array}\right\} \quad|q|<1 .
$$

In our proofs we follow some ideas employed by Andrews [1]. 


\section{The first general theorem}

THEOREM 2.1. Let $C_{k}(n)$ be the number of partitions of $n$ into distinct parts of the form $n=a_{1}+\cdots+a_{s}$ such that $a_{s} \equiv(k+1)$ or $(k+2)(\bmod 4)$ with $a_{s} \geq k+1, a_{j} \equiv(k+1)$ or $(k+2)(\bmod 4)$ if $a_{j+1} \equiv(k+2)$ or $(k+3)(\bmod 4)$, and $a_{j} \equiv k$ or $(k+3)(\bmod 4)$ if $a_{j+1} \equiv k$ or $(k+1)(\bmod 4)$. Then, for $k \geq 0$,

$$
\sum_{n=0}^{\infty} C_{k}(n) q^{n}=\sum_{n=0}^{\infty} \frac{\left(-q ; q^{2}\right)_{n} q^{n^{2}+k n}}{\left(q^{4} ; q^{4}\right)_{n}}
$$

Proof. We define $f(s, n)$ as the number of partitions of the type enumerated by $C_{k}(n)$ with the added restriction that the number of parts is exactly $s$. The following identity is true for $f(s, n)$ :

$$
f(s, n)=f(s-1, n-2 s-k+1)+f(s-1, n-4 s-k+2)+f(s, n-4 s) .
$$

To prove this we split the partitions enumerated by $f(s, n)$ into three classes: (a) those in which $k+1$ is a part, (b) those in which $k+2$ is a part and (c) those with all parts greater than $k+2$.

If in those in class (a) we drop the part $k+1$ and subtract 2 from each of the remaining parts we are left with a partition of $n-(k+1)-2(s-1)=n-2 s-k+1$ in exactly $s-1$ parts each greater than or equal to $k+1$ and these are the ones enumerated by $f(s-1, n-2 s-k+1)$. From those in class (b) we drop the part $k+2$ and subtract 4 from each of the remaining parts obtaining partitions that are enumerated by $f(s-1, n-4 s-k+2)$ and for the ones in class (c) we subtract 4 from each part obtaining the partitions enumerated by $f(s, n-4 s)$.

Defining

$$
F(z, q)=\sum_{n=0}^{\infty} \sum_{s=0}^{\infty} f(s, n) z^{s} q^{n}
$$

and using (2.2) we obtain

$$
\begin{aligned}
F(z, q)= & \sum_{n=0}^{\infty} \sum_{s=0}^{\infty}(f(s-1, n-2 s-k+1)+f(s-1, n-4 s-k+2)+f(s, n-4 s)) z^{s} q^{n} \\
= & z q^{k+1} \sum_{n=0}^{\infty} \sum_{s=0}^{\infty} f(s-1, n-2 s-k+1)\left(z q^{2}\right)^{s-1} q^{n-2 s-k+1} \\
& +z q^{k+2} \sum_{n=0}^{\infty} \sum_{s=0}^{\infty} f(s-1, n-4 s-k+2)\left(z q^{4}\right)^{s-1} q^{n-4 s-k+2} \\
& +\sum_{n=0}^{\infty} \sum_{s=0}^{\infty} f(s, n-4 s)\left(z q^{4}\right)^{s} q^{n-4 s} \\
= & z q^{k+1} F\left(z q^{2}, q\right)+z q^{k+2} F\left(z q^{4}, q\right)+F\left(z q^{4}, q\right) .
\end{aligned}
$$

If $F(z, q)=\sum_{n=0}^{\infty} \gamma_{n} z^{n}$ we may compare coefficients of $z^{n}$ in (2.4) obtaining

$$
\gamma_{n}=\gamma_{n-1} q^{2 n+k-1}+\gamma_{n-1} q^{4 n+k-2}+\gamma_{n} q^{4 n} .
$$


Therefore

$$
\gamma_{n}=q^{2 n+k-1} \frac{\left(1+q^{2 n-1}\right)}{\left(1-q^{4 n}\right)} \gamma_{n-1}
$$

and observing that $\gamma_{0}=1$ we may iterate (2.6) to get

$$
\gamma_{n}=\frac{\left(-q ; q^{2}\right)_{n} q^{n^{2}+k n}}{\left(q^{4} ; q^{4}\right)_{n}} .
$$

From this

$$
F(z, q)=\sum_{n=0}^{\infty} \frac{\left(-q ; q^{2}\right)_{n} q^{n^{2}+k n} z^{n}}{\left(q^{4} ; q^{4}\right)_{n}}
$$

and therefore

$$
\sum_{n=0}^{\infty} C_{k}(n) q^{n}=\sum_{n=0}^{\infty} \sum_{s=0}^{\infty} f(s, n) q^{n}=F(1, q)=\sum_{n=0}^{\infty} \frac{\left(-q ; q^{2}\right)_{n} q^{n^{2}+k n}}{\left(q^{4} ; q^{4}\right)_{n}} .
$$

If we consider $k=0$ in this theorem and denote $C_{0}(n)$ by $C(n)$ we have that $C(n)$ is the number of partitions of $n$ into distinct parts of the form $n=a_{1}+a_{2}+\cdots+a_{s}$, where $a_{s} \equiv 1$ or $2(\bmod 4), a_{j} \equiv 3$ or $4(\bmod 4)$ if $a_{j+1} \equiv 1$ or $4(\bmod 4)$ and $a_{j} \equiv 1$ or $2(\bmod 4)$ if $a_{j+1} \equiv 2$ or $3(\bmod 4)$.

If we let $D(n)$ denote the number of partitions of $n$ into even parts $\equiv \pm 4(\bmod 12)$ and distinct odd parts $\equiv \pm 5(\bmod 12)$ and let $E(n)$ denote the number of partitions of $n$ into even parts $\equiv \pm 4(\bmod 12)$ and distinct odd parts $\equiv \pm 1(\bmod 12)$, then we have the following theorem.

THEOREM 2.2. $C(n)=D(n)+E(n-1)$ for every positive integer $n$.

Proof. We have

$$
\begin{aligned}
\sum_{n=0}^{\infty} C(n) q^{n}= & \sum_{n=0}^{\infty} \frac{\left(-q ; q^{2}\right)_{n} q^{n^{2}}}{\left(q^{4} ; q^{4}\right)_{n}} \\
= & \sum_{n=0}^{\infty} \frac{\left(-q ; q^{2}\right)_{2 n} q^{4 n^{2}}}{\left(q^{4} ; q^{4}\right)_{2 n}}+q \sum_{n=0}^{\infty} \frac{\left(-q ; q^{2}\right)_{2 n+1} q^{4 n^{2}+4 n}}{\left(q^{4} ; q^{4}\right)_{2 n+1}} \\
= & \prod_{n=1}^{\infty} \frac{\left(1+q^{12 n-5}\right)\left(1+q^{12 n-7}\right)\left(1-q^{12 n}\right)}{\left(1-q^{4 n}\right)} \\
& +q \prod_{n=1}^{\infty} \frac{\left(1+q^{12 n-1}\right)\left(1+q^{12 n-11}\right)\left(1-q^{12 n}\right)}{\left(1-q^{4 n}\right)} \\
= & \sum_{n=0}^{\infty} D(n) q^{n}+q \sum_{n=0}^{\infty} E(n) q^{n},
\end{aligned}
$$

where we have used identities (1.4) and (1.5) after replacing in (1.4) " $q$ " by “- $q$ " which completes the proof. 
We list below the partitions of 21 enumerated by $C(21)$ and $D(21)$ and the ones of 20 enumerated by $E(20)$.

It is interesting to observe that

$$
C(n)=F_{e}(n)-F_{0}(n)
$$

where $F_{e}(n)$ (respectively, $\left.F_{0}(n)\right)$ is the number of partitions of $n$ into parts $\not \equiv 0$, $\pm 2(\bmod 12)$ with no repeated multiples of 3 and with an even (respectively, odd) number of parts divisible by 3 .

TABLE 2.1.

\begin{tabular}{|l|l|l|}
\hline$C(21)=12$ & $D(21)=5$ & $E(20)=7$ \\
\hline 21 & $17+4$ & 20 \\
$20+1$ & $16+5$ & $16+4$ \\
$12+8+1$ & $8+8+5$ & $8+8+4$ \\
$16+5$ & $8+5+4+4$ & $8+4+4+4$ \\
$12+9$ & $5+4+4+4+4$ & $4+4+4+4+4$ \\
$17+3+1$ & & $11+8+1$ \\
$16+4+1$ & & $11+4+4+1$ \\
$13+7+1$ & & \\
$13+6+2$ & & \\
$9+7+5$ & & \\
$12+5+3+1$ & & \\
$9+7+4+1$ & & \\
\hline
\end{tabular}

In fact, by replacing " $q$ " by " $-q$ " in (1.3) we have

$$
\begin{array}{r}
\prod_{n=1}^{\infty}\left(1+q^{12 n-5}\right)\left(1+q^{12 n-7}\right)\left(1-q^{12 n}\right)+q \prod_{n=1}^{\infty}\left(1+q^{12 n-1}\right)\left(1+q^{12 n-11}\right)\left(1-q^{12 n}\right) \\
=\prod_{n=1}^{\infty}\left(1+q^{3 n-1}\right)\left(1+q^{3 n-2}\right)\left(1-q^{3 n}\right)
\end{array}
$$

and observing that, by (2.10),

$$
\begin{aligned}
\sum_{n=0}^{\infty} C(n) q^{n} & =\prod_{n=1}^{\infty} \frac{\left(1+q^{3 n-1}\right)\left(1+q^{3 n-2}\right)\left(1-q^{3 n}\right)}{\left(1-q^{4 n}\right)} \\
& =\frac{\prod_{\substack{n=1 \\
n \neq 0(\bmod 4)}}^{\infty}\left(1-q^{3 n}\right)}{\prod_{\substack{n=1 \\
n \equiv \pm 1, \pm 4, \pm 5(\bmod 12)}}^{\infty}\left(1-q^{n}\right)}=\sum_{n=0}^{\infty}\left(F_{e}(n)-F_{0}(n)\right) q^{n}
\end{aligned}
$$

we have (2.11).

Next, we state and prove our second general result. 


\section{The second general theorem}

THEOREM 3.1. For $\ell \geq 0$ let $A_{\ell}(n)$ be the number of partitions of $n$ of the form $n=a_{1}+a_{2}+\cdots+a_{2 s-1}+a_{2 s}$ such that $a_{2 s} \geq a$ (respectively, $\left.a+1\right)$, where $\ell=$ $2 a$ (respectively, $2 a+1), a_{2 i-1}-a_{2 i}=1$ or 2 (respectively, 0 or 1 and $a_{2 i}>a_{2 i+1}$ ). Then

$$
\sum_{n=0}^{\infty} A_{\ell}(n) q^{n}=\sum_{n=0}^{\infty} \frac{\left(-q ; q^{2}\right)_{n} q^{n^{2}+\ell n}}{\left(q^{2} ; q^{2}\right)_{n}} .
$$

Proof. We define, for $\lambda=1$ or $2, g_{\lambda}(2 s, n)$ as the number of partitions of the type enumerated by $A_{\ell}(n)$ with the added restriction that the number of parts is exactly $2 s$ and such that $a_{2 s-1}+a_{2 s} \geq \ell+2 \lambda-1$. For $n=s=0$, we define $g_{\lambda}(2 s, n)=1$ and $g_{\lambda}(2 s, n)=0$ if $n<0$ or $s<0$ or $n=0$ and $s>0$.

In what follows we prove two identities for $g_{\lambda}(2 s, n)$.

(i) $g_{1}(2 s, n)=g_{2}(2 s-2, n-2 s-\ell)+g_{1}(2 s-2, n-2 s-\ell+1)+g_{1}(2 s, n-2 s)$,

(ii) $g_{2}(2 s, n)=g_{1}(2 s, n-2 s)$.

To prove the first one we split the partitions enumerated by $g_{1}(2 s, n)$ into two classes: (a) those partitions in which $\lfloor(\ell+1) / 2\rfloor$ is a part; (b) those in which $\lfloor(\ell+1) / 2\rfloor$ is not a part.

The ones in class (a) can have as the two smallest parts either " $\lfloor(\ell+4) / 2\rfloor+\lfloor(\ell+1) / 2\rfloor$ " or " $\lfloor(\ell+2) / 2\rfloor+\lfloor(\ell+1) / 2\rfloor$." In the first case, if we remove the two smallest parts and subtract 1 from each of the remaining parts we are left with a partition of $n-(\ell+2)-$ $(2 s-2)=n-2 s-\ell$ into exactly $2 s-2$ parts where $a_{2 s-3}+a_{2 s-2} \geq \ell+3$. These are the partitions enumerated by $g_{2}(2 s-2, n-2 s-\ell)$.

From those in class (a) having " $\lfloor(\ell+2) / 2\rfloor+\lfloor(\ell+1) / 2\rfloor$ " as the two smallest parts we remove these two and subtract 1 from each of the remaining parts. We are, in this case, left with a partition of $n-(\ell+1)-(2 s-2)=n-2 s-\ell+1$ into exactly $2 s-2$ parts where $a_{2 s-3}+a_{2 s-2} \geq \ell+1$ which are enumerated by $g_{1}(2 s-2, n-2 s-\ell+1)$.

It is important to observe that after doing these operations the restrictions on difference between parts is not changed.

Now we consider the partitions in class (b). Considering that $\lfloor(\ell+1) / 2\rfloor$ is not a part we can subtract 1 from each part obtaining partitions of $n-2 s$ into $2 s$ parts which are the ones enumerated by $g_{1}(2 s, n-2 s)$.

The proof of (ii) follows from the fact that if we subtract 1 from each part of a partition such that $a_{2 s-1}+a_{2 s} \geq \ell+3$ the resulting one is such that $a_{2 s-1}^{\prime}+a_{2 s}^{\prime} \geq \ell+1$.

Now we define

$$
G_{\lambda}(z, q)=\sum_{n=0}^{\infty} \sum_{s=0}^{\infty} g_{\lambda}(2 s, n) z^{2 s} q^{n} .
$$

Using (i) we have

$$
\begin{aligned}
G_{1}(z, q) & =\sum_{n=0}^{\infty} \sum_{s=0}^{\infty} g_{1}(2 s, n) z^{2 s} q^{n} \\
& =\sum_{n=0}^{\infty} \sum_{s=0}^{\infty}\left(g_{2}(2 s-2, n-2 s-\ell)+g_{1}(2 s-2, n-2 s-\ell+1)+g_{1}(2 s, n-2 s)\right) z^{2 s} q^{n}
\end{aligned}
$$




$$
\begin{aligned}
= & z^{2} q^{\ell+2} \sum_{n=0}^{\infty} \sum_{s=0}^{\infty} g_{1}(2 s-2, n-4 s-\ell+2)\left(z q^{2}\right)^{2 s-2} q^{n-4 s-\ell+2} \\
& +z^{2} q^{\ell+1} \sum_{n=0}^{\infty} \sum_{s=0}^{\infty} g_{1}(2 s-2, n-2 s-\ell+1)(z q)^{2 s-2} q^{n-2 s-\ell+1} \\
& +\sum_{n=0}^{\infty} \sum_{s=0}^{\infty} g_{1}(2 s, n-2 s)(z q)^{2 s} q^{n-2 s} \\
= & z^{2} q^{\ell+2} G_{1}\left(z q^{2}, q\right)+z^{2} q^{\ell+1} G_{1}(z q, q)+G_{1}(z q, q),
\end{aligned}
$$

where on the first term of the sum, we used (ii).

Now, comparing the coefficients of $z^{2 n}$ in (3.3) after making the substitution

$$
G_{1}(z, q)=\sum_{n=0}^{\infty} \gamma_{n} z^{2 n}
$$

we have

$$
\gamma_{n}=q^{4 n+\ell-2} \gamma_{n-1}+q^{2 n+\ell-1} \gamma_{n-1}+q^{2 n} \gamma_{n}
$$

Therefore

$$
\gamma_{n}=q^{2 n+\ell-1} \frac{\left(1+q^{2 n-1}\right)}{\left(1-q^{2 n}\right)} \gamma_{n-1}
$$

and, observing that $\gamma_{0}=1$, we may iterate this $n-1$ times to get:

$$
\gamma_{n}=\frac{\left(-q ; q^{2}\right)_{n} q^{n^{2}+\ell n}}{\left(q^{2} ; q^{2}\right)_{n}} .
$$

Then

$$
G_{1}(z, q)=\sum_{n=0}^{\infty} \frac{\left(-q ; q^{2}\right)_{n} q^{n^{2}+\ell n}}{\left(q^{2} ; q^{2}\right)_{n}} z^{2 n}
$$

and the theorem follows since

$$
\sum_{n=0}^{\infty} A_{\ell}(n) q^{n}=\sum_{n=0}^{\infty} \sum_{s=0}^{\infty} g_{1}(2 s, n) q^{n}=G_{1}(1, q)=\sum_{n=0}^{\infty} \frac{\left(-q ; q^{2}\right)_{n} q^{n^{2}+\ell n}}{\left(q^{2} ; q^{2}\right)_{n}}
$$

Particular CASeS of TheOrem 3.1. If we take $\ell=0$ in Theorem 3.1 we have the following result.

THEOREM 3.2. The number of partitions of $n$ into an even number of parts, $2 s$, such that $a_{2 j-1}-a_{2 j}=1$ or 2 and $a_{2 s} \geq 0$ is equal to the number of partitions of $n$ into parts $\equiv \pm 1,4(\bmod 8)$.

Proof. By Theorem 3.1 and (1.2), we have

$$
\sum_{n=0}^{\infty} A_{0}(n) q^{n}=\sum_{n=0}^{\infty} \frac{\left(-q ; q^{2}\right)_{n} q^{n^{2}}}{\left(q^{2} ; q^{2}\right)_{n}}=\prod_{n=1}^{\infty} \frac{1}{\left(1-q^{8 n-1}\right)\left(1-q^{8 n-4}\right)\left(1-q^{8 n-7}\right)} .
$$


For $\ell=1$ we have the following theorem.

THEOREM 3.3. $A_{1}(n)$ is equal to the number of partitions of $n$ into parts $\equiv 2,3$, $7(\bmod 8)$.

Proof. By Andrews [2, Theorem 3 and Corollary 2.7, page 21] with $q$ replaced by $q^{2}$ and after $a=-q$, we have

$$
\begin{aligned}
\sum_{n=0}^{\infty} A_{1}(n) q^{n} & =\sum_{n=0}^{\infty} \frac{\left(-q ; q^{2}\right)_{n} q^{n(n+1)}}{\left(q^{2} ; q^{2}\right)_{n}}=\left(-q^{3} ; q^{4}\right)_{\infty}\left(-q^{2} ; q^{2}\right)_{\infty} \\
& =\prod_{n=1}^{\infty} \frac{1}{\left(1-q^{8 n-1}\right)\left(1-q^{8 n-5}\right)\left(1-q^{8 n-6}\right)} .
\end{aligned}
$$

In [4], Santos and Mondek gave a family of partitions including as a special case the following theorem of Göllnitz.

"Let $G_{2}(n)$ denote the number of partitions of $n$ into parts, where each part is congruent to one of 2,3 or $7(\bmod 8)$. Let $H_{2}(n)$ denote the number of partitions of $n$ of the form $b_{1}+b_{2}+\cdots+b_{j}$, where $b_{i} \geq b_{i+1}+2$ and strict inequality holds if $b_{i}$ is odd; in addition, $b_{j} \geq 2$. Then for each $n, G_{2}(n)=H_{2}(n)$."

It is clear that by our Theorem 3.3 we have a new combinatorial interpretation for partitions enumerated by $\mathrm{H}_{2}(n)$.

For $\ell=2$ we get the following result.

THEOREM 3.4. The number of partitions of $n$ into an even number of parts, $2 s$, such that $a_{2 j-1}-a_{2 j}=1$ or 2 , and $a_{2 s} \geq 1$, is equal to the number of partitions of $n$ into parts $\equiv \pm 3,4(\bmod 8)$.

Proof. By Theorem 3.1 and (1.1), we have

$$
\sum_{n=0}^{\infty} A_{2}(n) q^{n}=\sum_{n=0}^{\infty} \frac{\left(-q ; q^{2}\right)_{n} q^{n^{2}+2 n}}{\left(q^{2} ; q^{2}\right)_{n}}=\prod_{n=1}^{\infty} \frac{1}{\left(1-q^{8 n-3}\right)\left(1-q^{8 n-4}\right)\left(1-q^{8 n-5}\right)}
$$

4. A bijection. We now describe a transformation between the partitions defined by $A_{2}(n)$ and partitions of $n$ into $s$ parts.

The partitions of $n$ with an even number of parts $2 s$ where $a_{2 j-1}-a_{2 j}=1$ or 2 , $a_{2 s} \geq 1$ can be transformed into partitions of $n$ into $s$ parts just by adding the parts $a_{2 j-1}+a_{2 j}$, i.e., the partition

$$
a_{1}+a_{2}+\cdots+a_{2 j-1}+a_{2 j}+a_{2 j+1}+\cdots+a_{2 s}
$$

is transformed into

$$
b_{1}+b_{2}+\cdots+b_{j}+\cdots+b_{s}
$$

where $b_{j}=a_{2 j-1}+a_{2 j}, b_{j}-b_{j+1} \geq 2$ and $b_{j}-b_{j+1} \geq 3$ if $b_{j+1}$ is even with the restriction $b_{s} \geq 3$. 
TABLE 4.2 .

\begin{tabular}{|c|c|c|}
\hline 16 & $\longleftrightarrow$ & $9+7$ \\
\hline $13+3$ & $\longleftrightarrow$ & $7+6+2+1$ \\
\hline $12+4$ & $\longleftrightarrow$ & $7+5+3+1$ \\
\hline $11+5$ & $\longleftrightarrow$ & $6+5+3+2$ \\
\hline $10+6$ & $\longleftrightarrow$ & $6+4+4+2$ \\
\hline $9+7$ & $\longleftrightarrow$ & $5+4+4+3$ \\
\hline $8+5+3$ & $\rightleftarrows$ & $5+3+3+2+2+1$ \\
\hline
\end{tabular}

This operation can be easily reversed in the following way:

- If $b_{j}$ is even we write it as $a_{2 j-1}+a_{2 j}$, where $a_{2 j-1}=\left(b_{j} / 2\right)+1$ and $a_{2 j}=\left(b_{j} / 2\right)-1$.

- If $b_{j}$ is odd we write it as $a_{2 j-1}+a_{2 j}$, where $a_{2 j-1}=\left(b_{j}+1\right) / 2$ and $a_{2 j}=\left(b_{j}-1\right) / 2$. With this transformation we get the original one

$$
a_{1}+a_{2}+\cdots+a_{2 s}
$$

with exactly the same restrictions, i.e., $a_{2 j-1}-a_{2 j}=1$ or 2 and $a_{2 s} \geq 1$.

To illustrate this we list the partitions of 16 as described in Theorem 3.4 and the ones obtained by the transformation given above.

Theorem 4.1 follows from this transformation and (1.1).

THEOREM 4.1. The number of partitions of $n$ of the form $n=b_{1}+b_{2}+\cdots+b_{s}$, where $b_{j}-b_{j+1} \geq 2, b_{s} \geq 3$, and $b_{j}-b_{j+1} \geq 3$ if $b_{j+1}$ is even, is equal to the number of partitions of $n$ into parts $\equiv \pm 3,4(\bmod 8)$.

This Theorem was proved by Gordon in [3, Theorem 3, page 741].

Also by Theorem 3.2 and the bijection described we have the following theorem.

THEOREM 4.2. The number of partitions of any positive integer $n$ into parts $\equiv 1,4$ or $7(\bmod 8)$ is equal to the number of partitions of the form $n=n_{1}+n_{2}+\cdots+n_{k}$, where $n_{i} \geq n_{i+1}+2$, and $n_{i} \geq n_{i+1}+3$ if $n_{i}$ is even $(1 \leq i \leq k-1)$.

This result has been proved by Gordon in [3, Theorem 2, page 741].

ACKNOWLEDGEMENTS. We would like to thank the referee for suggesting the interpretation for our $C(n)$ given in (2.11) and also for many corrections. The work of Santos was partially supported by FAPESP.

\section{REFERENCES}

[1] G. E. Andrews, Applications of basic hypergeometric functions, SIAM Rev. 16 (1974), 441484. MR 50\#5044. Zbl 299.33004.

[2] - The Theory of Partitions, Cambridge Mathematical Library, Cambridge University Press, Cambridge, 1998, Reprint of the 1976 original. MR 99c:11126. Zbl 980.49233.

[3] B. Gordon, Some continued fractions of the Rogers-Ramanujan type, Duke Math. J. 32 (1965), 741-748. MR 32\#1477. Zbl 178.33404.

[4] J. P. de O. Santos and P. Mondek, Extending theorems of Göllnitz, a new family of partition identities, Ramanujan J. 3 (1999), no. 4, 359-365. CMP 1738 902. Zbl 991.19548. 
[5] L. J. Slater, Further identities of the Rogers-Ramanujan type, Proc. London Math. Soc. (2) 54 (1952), 147-167. MR 14,138e. Zbl 046.27204.

JosÉ PLÍNIo O. SANTOS: IMECC-UNICAMP CX.P. 6065, 13083-970, CAMPINAS, SP, BRASIL

E-mail address: josep1i@ime.unicamp.br, santos@math.psu.edu

PAUlo MoNDEK: CCET-UFMS CX.P. 549, 79070-900, CAMPO GRANDE, MS, BRASIL

E-mail address: mondek@hi 1bert.dmt.ufms.br 


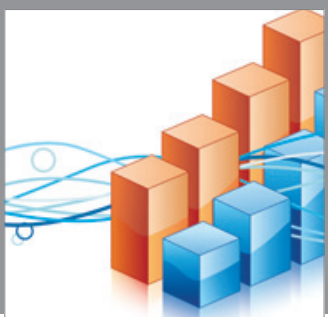

Advances in

Operations Research

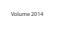

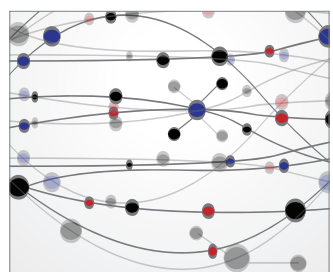

\section{The Scientific} World Journal
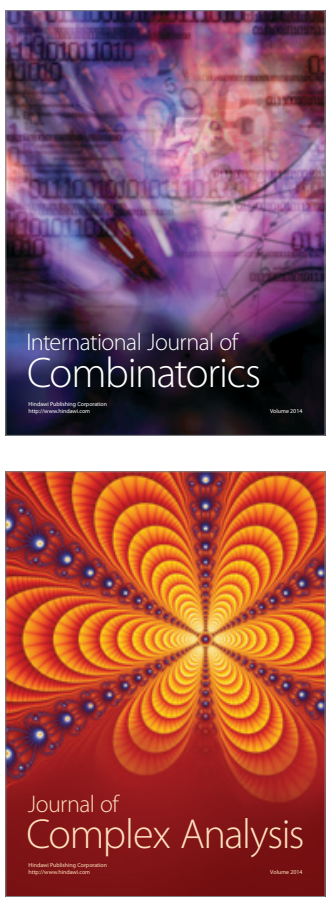

International Journal of

Mathematics and

Mathematical

Sciences
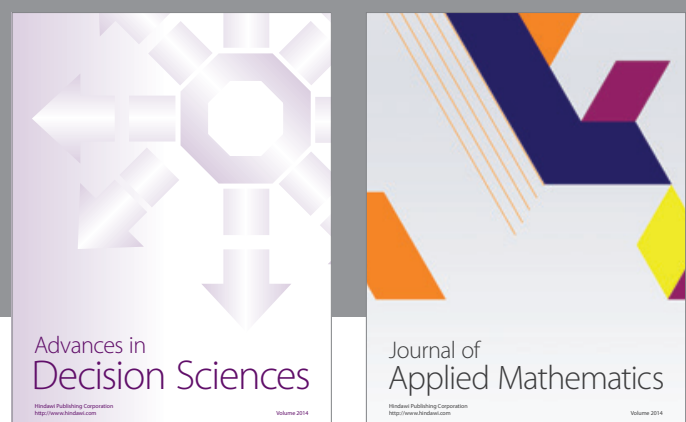

Journal of

Applied Mathematics
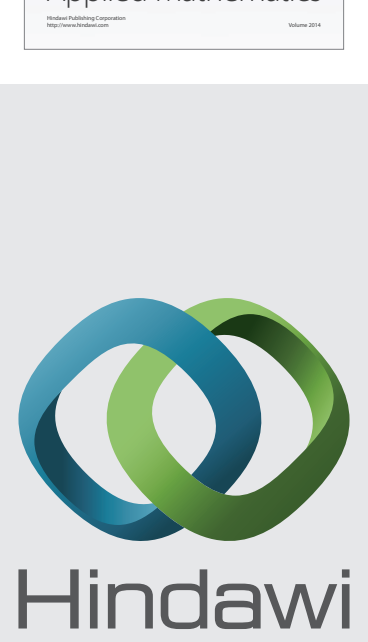

Submit your manuscripts at http://www.hindawi.com
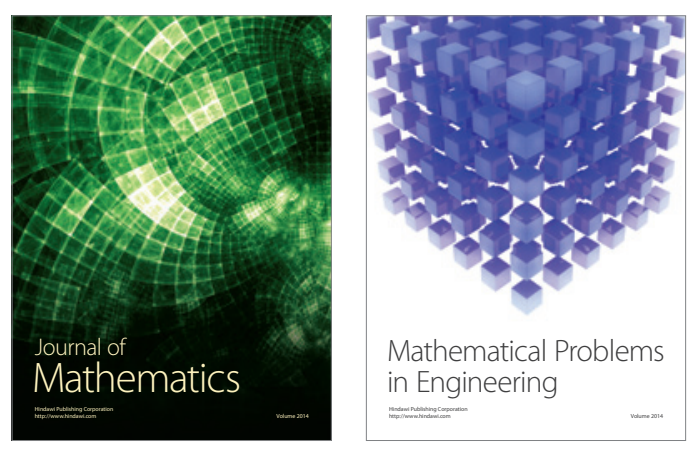

Mathematical Problems in Engineering
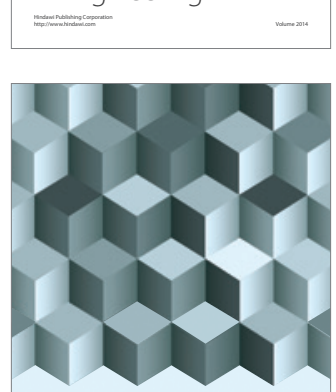

Journal of

Function Spaces
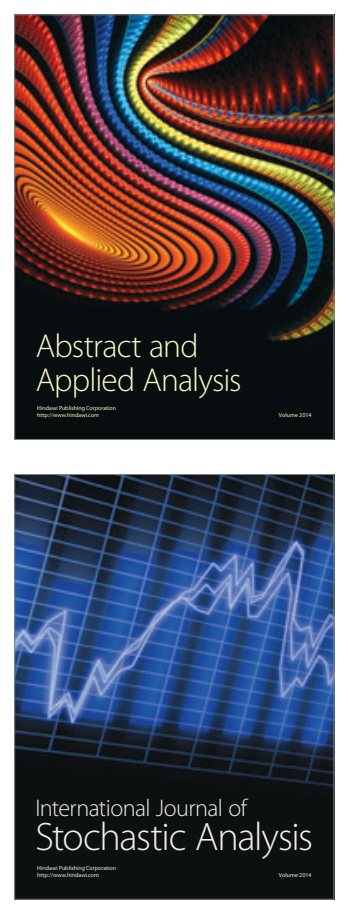

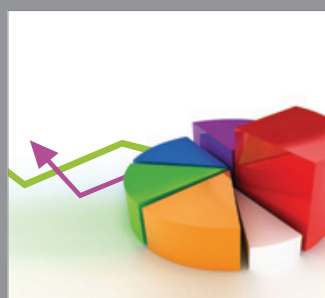

ournal of

Probability and Statistics

Promensencen
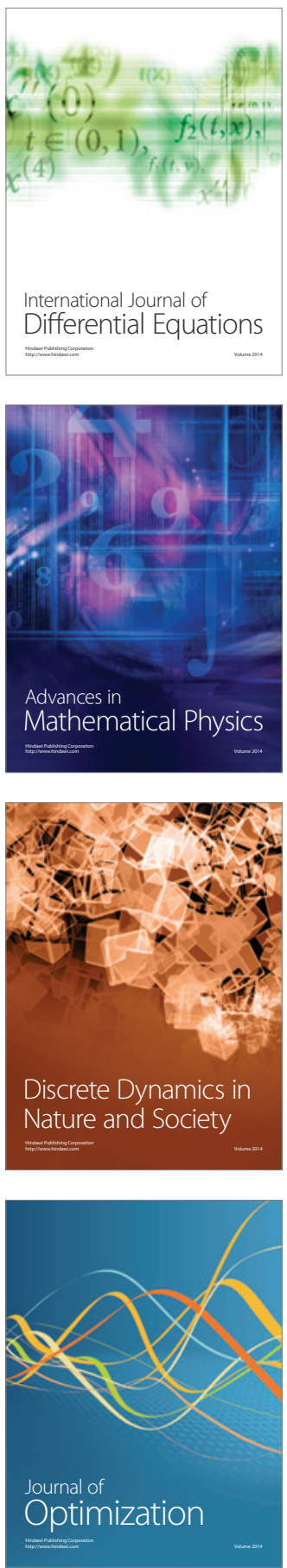